\title{
Monitorização Hemodinâmica em Cirurgia Cardíaca Pediátrica
}

\author{
Fernando Antibas Atik
}

São Paulo, SP

A cirurgia cardíaca pediátrica é considerada uma das subespecialidades mais complexas dentro da medicina. $\mathrm{O}$ conhecimento acumulado nas últimas décadas pelo entendimento da fisiopatologia das diversas cardiopatias congênitas, aliado aos progressos tecnológicos incorporados à prática médica, tem permitido a correção cirúrgica de grande parte das anomalias cardíacas, com redução significativa dos índices de morbidade e mortalidade. Contribuem para tal a melhoria na proteção miocárdica e cerebral, na técnica cirúrgica, no cuidado peri-operatório e nas técnicas de circulação extracorpórea. Muitas das cardiopatias congênitas consideradas inoperáveis no passado, como a hipoplasia do coração esquerdo, são tratadas cirurgicamente no período neonatal, com resultados promissores ${ }^{1}$.

No entanto, apesar de todos os avanços alcançados, a morbidade e a mortalidade encontradas em alguns centros, especialmente em relação à cirurgia realizada durante o período neonatal, não são desprezíveis. Grande parte desse insucesso reside na grande complexidade desse grupo de pacientes, porém, a falha do reconhecimento precoce e adequado da hipoperfusão tecidual, talvez seja o maior fator. A grande gama de cardiopatias congênitas com mecanismos fisiopatológicos distintos, aliada à pobreza de sinais clínicos característicos, exige das pessoas envolvidas no atendimento, maior conhecimento e experiência clínica. Somente a suspeita clínica pode desencadear a investigação diagnóstica complementar e o tratamento apropriado. O respeito ao conceito do atendimento multiprofissional é de extrema valia na manutenção e eficiência desse sistema ${ }^{2}$.

A monitorização hemodinâmica desempenha papel fundamental neste sentido, e deve atender aos modernos conceitos de choque, cuja base reside no metabolismo celular. Desta forma, a monitorização hemodinâmica atual busca

Cleveland Clinic Foundation Cleveland, OHIO - USA - Pediatric Cardiac Surgery Endereço para Correspondência: Dr. Fernando Antibas Atik - Av. Chibarás, 626/101 Cep 04076-003 - São Paulo, SP - E-mail: fernandoatik@aol.com Recebido para publicação em 27/2/03

Aceito em 11/8/03 a detecção precoce do desbalanço entre oferta e consumo de oxigênio $\left(\mathrm{O}_{2}\right)$, podendo definir preditores de sobrevida e incrementar a estratificação de risco, com especial importância na estimativa do prognóstico.

Neste contexto estimativo os parâmetros clínicos incluem, principalmente, os pulsos periféricos, o enchimento capilar, a frequência cardíaca, a pressão arterial, o nível de consciência e a diurese. Estas variáveis mostraram-se ineficazes na detecção de alterações precoces do estado hemodinâmico, pois a exteriorização clínica ocorre quando há exaustão dos mecanismos compensatórios do organismo, que priorizam a manutenção da pressão arterial. A hipotensão arterial é um sinal tardio e pouco confiável do baixo débito cardíaco ${ }^{3}$, e a normalização de seus valores não é o objetivo da ressuscitação dos estados de choque ${ }^{4}$. A temperatura dos membros inferiores tem sido utilizada como uma das medidas de perfusão periférica em crianças após cirurgia cardíaca, e a grande diferença entre essa medida e a temperatura central foi proposta como preditor de mortalidade pós-operatória, porém sem validação por alguns estudos ${ }^{5}$.

Em cirurgia cardíaca pediátrica, a monitorização hemodinâmica padrão inclui o eletrocardiograma, a pressão arterial invasiva, a pressão venosa central, a oximetria de pulso e a capnografia ${ }^{6}$. Os empregos da monitorização complementar por meio das pressões do átrio esquerdo e/ou da artéria pulmonar e da ecocardiografia seriada no pós-operatório, devem ser incorporados especialmente nos neonatos, em operações corretivas ou paliativas complexas, e naqueles com suspeita de hipertensão pulmonar. A monitorização contínua da pressão da artéria pulmonar tem se mostrado fundamental no manejo pós-operatório da drenagem anômala total de veias pulmonares ${ }^{7}$ e de outras cardiopatias complexas.

Entretanto, a definição de marcadores confiáveis de hipoperfusão tecidual tem sido extremamente difícil de ser alcançada. O desenvolvimento de preditores de mortalidade, envolve o conhecimento de múltiplos índices fisiológi$\cos _{\text {distintos }}^{8-11}$. A dificuldade em determinar esses índices ocorre freqüentemente nos portadores de cardiopatias congênitas, pela limitação da monitorização invasiva e de sua confiabilidade, impostas pelo peso do paciente. Além disso, 
certas características hemodinâmicas, como o desvio de sangue no plano atrial, podem interferir na mensuração do débito cardíaco, da saturação venosa de $\mathrm{O}_{2}$ e da oferta e consumo de $\mathrm{O}_{2}$.

Uma série de problemas metodológicos ${ }^{12}$ tem sido identificada nos diversos estudos que analisam fatores de risco de morbidade e mortalidade em cirurgia cardíaca pediátrica. Em primeiro lugar, existe carência de estudos prospectivos randomizados com grandes populações. A dificuldade de executar um estudo dessa natureza reside na falta de normatização na definição dos fatores mórbidos e da insuficiência de múltiplos órgãos e sistemas, pois as classificações divergem. Um outro problema é a baixa incidência de complicações pós-operatórias e de óbito na maioria dos estudos, devido a realização de operações mais simples, ou pelos resultados cirúrgicos excelentes, o que torna as estatísticas pouco significativas. A maioria dos estudos analisa somente o período pós-operatório, não refletindo os problemas que ocorrem durante a circulação extracorpórea.

O objetivo desta atualização é revisar as diversas modalidades de monitorização hemodinâmica em cirurgia cardíaca pediátrica através de revisão bibliográfica detalhada, aliada à experiência pessoal no atendimento a pacientes no pós-operatório de cirurgia cardíaca pediátrica.

Os efeitos da circulação extra-corpórea. A criança nãoé um adulto pequeno - Muitas diferenças entre crianças e adultos afetam a resposta à circulação extracorpórea, e devem ser consideradas nas estratégias de perfusão durante a cirurgia cardíaca ${ }^{13}$. Assim, a criança apresenta menor volemia, maior consumo de oxigênio, maior reatividade vascular pulmonar, presença de shunts intra e extracardíacos, sistemas orgânicos imaturos, alterações na termorregulação e baixa tolerância à embolia.

O choque é caracterizado pelo desbalanço entre oferta e consumo de $\mathrm{O}_{2}$, com manutenção do metabolismo anaeróbico. A adequação da perfusão tecidual e seus mecanismos adaptativos, já foi bem estudada em adultos. Os mecanismos gerais não diferem na criança, porém existem algumas peculiaridades que devem ser examinadas. Primeiramente, o consumo de $\mathrm{O}_{2}$ na criança em repouso é maior que nos adul$\operatorname{tos}^{14}$. A hipotermia profunda e a parada circulatória total são empregadas mais freqüentemente na cirurgia cardíaca pediátrica, tendo efeitos nas relações entre oferta e consumo de $\mathrm{O}_{2}$. Além disso, a resposta metabólica ao stress na criança é conhecidamente mais exuberante que no adulto ${ }^{15}$.

O consumo de $\mathrm{O}_{2}$ é independente da oferta numa grande variedade de situações. Durante períodos de diminuição da oferta de $\mathrm{O}_{2}$, o metabolismo aeróbico é mantido inicialmente às custas de maior extração de $\mathrm{O}_{2}$ pelos tecidos. Com esse mecanismo o consumo se torna independente da oferta, até o ponto crítico ser atingido. Nesse ponto, que corresponde a uma taxa de extração por volta de 50 a $60 \%$, o consumo torna-se dependente da oferta de $\mathrm{O}_{2}$ e ocorre a acidose láctica $^{16-17}$ (fig. 1).

Um desbalanço entre oferta e consumo pode ocorrer regionalmente, mas o fluxo sanguíneo regional é difícil de se monitorar durante a circulação extracorpórea. Nesta, são

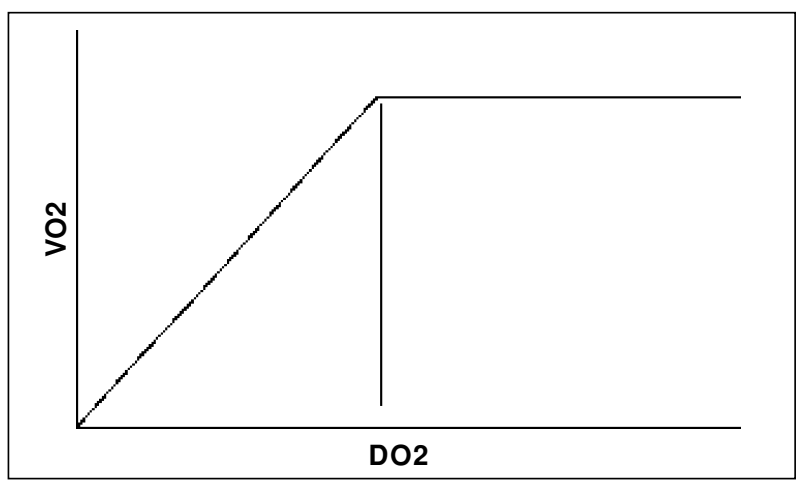

Fig. 1- Relação entre oferta $\left(\mathrm{DO}_{2}\right)$ e consumo de oxigênio $\left(\mathrm{VO}_{2}\right)$ nos estados de choque. A curva ascendente representa a fase patológica, onde o consumo torna-se dependente da oferta de $\mathrm{O}_{2}$ e ocorre acidose láctica (mais detalhes no texto).

alterados o débito cardíaco, o tônus vasomotor de vários órgãos, e a patência da microcirculação. Além disso, os requerimentos metabólicos regionais podem estar aumentados devido ao esfriamento e aquecimento não homogêneos e como resultado da falha na oferta de $\mathrm{O}_{2}$ aos tecidos. A resposta inflamatória com liberação de citoquinas e a ativação dos hormônios de estresse que ocorrem durante a circulação extracorpórea, também aumentam as demandas metabólicas ${ }^{18,19}$.

Durante a circulação extracorpórea, o fluxo sanguíneo é significativamente alterado nos diversos sistemas orgânicos, tanto durante a fase normotérmica quanto hipotérmica, bem como nos fluxos altos ou baixos de perfusão ${ }^{20}$. O fluxo sanguíneo durante a fase hipotérmica por exemplo é desviado para o músculo esquelético, particularmente nos casos com altos fluxos de perfusão. Adicionalmente, o retorno do consumo de oxigênio de todo o organismo para o nível basal após o reaquecimento, não necessariamente se reflete regionalmente, já que os órgãos apresentam diferentes taxas de metabolismo celular.

No estudo da hipoperfusão regional, dois órgãos merecem destaque, o cérebro e o trato gastrointestinal. A criança é especialmente susceptível a complicações neurológicas secundárias à circulação extracorpórea, dentre elas as crises convulsivas, coreoatetose, acidente vascular cerebral e retardo de desenvolvimento psicomotor e intelectual. A etiologia é multifatorial ${ }^{13}$, mas pode envolver a macro e a microembolização, a hipotermia profunda, a parada circulatória total, o manejo dos gases arteriais ( $\mathrm{pH}$-stat versus alpha-stat), o tempo de esfriamento e reaquecimento e a perda da auto-regulação do fluxo sanguíneo cerebral.

A hipoperfusão mesentérica secundária à síndrome de baixo débito cardíaco resulta em maior permeabilidade intestinal, com translocação bacteriana e passagem de endotoxinas para a circulação sistêmica. O desencadeamento da resposta inflamatória sistêmica é resultado da ativação de citoquinas, como a interleucina-6 e o fator de necrose tumoral. A integridade do trato gastrointestinal pode também ser alterada durante a circulação extracorpórea, cujos efeitos sobre o fluxo sanguíneo esplâncnico têm demonstrado, que hipoperfusão significativa pode ocorrer ${ }^{21,22}$, sendo o meca- 
nismo, desencadeador de uma cascata de eventos que culminam com a insuficiência de múltiplos órgãos e sistemas.

Por que ocorre a hipoperfusão esplâncnica durante a circulação extracorpórea? Uma série de particularidades ocorre na cirurgia cardíaca que predispõe este tipo de complicação. Nos estados de choque, alguns mecanismos adaptativos do organismo são desencadeados a fim de priorizar a perfusão de órgãos considerados nobres, como o cérebro e o coração. Durante a circulação extracorpórea o predomínio de agentes vasoconstritores (catecolaminas, angiotensina II e vasopressina) sobre os vasodilatadores (histamina, prostaglandina $\mathrm{E}_{2}$ e bradicinina) leva à diminuição do fluxo sanguíneo esplâncnico. Defeitos anatômicos ou correções cirúrgicas estão associados a "roubo" do fluxo sistêmico, como ocorre nas cardiopatias congênitas dependentes do canal arterial quando há desbalanço entre as resistências vasculares sistêmica e pulmonar, ocorrido, especialmente, na presença de colaterais sistêmico-pulmonares, na atresia pulmonar com comunicação interventricular, nos pacientes com anastomoses sistêmico-pulmonares de grande calibre e no pós-operatório da cirurgia de Norwood. Finalmente, o edema da mucosa intestinal pode ocorrer durante a circulação extracorpórea, podendo limitar a perfusão intestinal e a extração de $\mathrm{O}_{2}$.

O grupo de pacientes mais susceptível a essas complicações no pós-operatório é o dos neonatos e as alterações desencadeadas durante a circulação extracorpórea exigem atenção especial, por uma série de motivos: 1) as operações são geralmente complexas e prolongadas, necessitando por vezes de hipotermia profunda e parada circulatória total;2) os efeitos da hemodiluição são mais pronunciados; 3 ) a redução do hematócrito e da pressão oncótica limita a oferta de $\mathrm{O}_{2}$ e a diminuição na resistência vascular sistêmica pode comprometer a perfusão tecidual; 4) maior exposição do sangue circulante à superfície não endotelial do circuito extracorpóreo, ocorrendo exacerbação da resposta inflamatória sistêmica, com liberação de citoquinas e radicais livres de $\mathrm{O}_{2}$, os quais podem lesar diretamente os tecidos ou alterar a microcirculação; 5) portadores de defeitos que cursam com fisiologia univentricular e são submetidos a operações paliativas são mais susceptíveis ao hiperfluxo pulmonar, à isquemia coronariana e ao baixo débito cardíaco, em consequência dos desbalanços entre as resistências vasculares sistêmica e pulmonar.

Não menos importantes a duração da circulação extracorpórea, da anóxia miocárdica, do grau de hipotermia, a duração do esfriamento e do aquecimento, a estratégia de manipulação do pH e o valor do hematócrito, são todos fatores potenciais que podem contribuir para a hipoperfusão durante sua realização.

Torna-se óbvia, a necessidade de entender os fatores predisponentes à hipoperfusão tecidual e é fundamental que a monitorização hemodinâmica seja incluída durante a cirurgia cardíaca e a circulação extracorpórea, fato que não ocorre, presentemente, na maioria dos centros.

Qual o fluxo ideal e como monitorar a perfusão tecidual durante a circulação extracorpórea? - Durante a circulação extracorpórea, o débito cardíaco é mantido pelo
Monitorização hemodinâmica em cirurgia cardíaca pediátrica

fluxo arterial de perfusão, uma variável calculada de acordo com a superfície corpórea e a taxa de metabolismo celular, diretamente relacionada à temperatura. O fluxo geralmente utilizadoé de $2 \mathrm{a} 2,4 \mathrm{~L} / \mathrm{m}^{2} / \mathrm{min}$ ou 100 a $120 \mathrm{~mL} / \mathrm{kg} / \mathrm{min}$, valores bem longe dos valores fisiológicos do índice cardíaco ${ }^{23}$. Neonatos e crianças pequenas requerem valores muito maiores que esses, para atender às necessidades metabólicas, sendo perfundidos com fluxos maiores que $200 \mathrm{~mL} / \mathrm{Kg} / \mathrm{min}$.

A hipotermia empregada durante a circulação extracorpórea permite que o fluxo de perfusão seja reduzido, dada à diminuição do metabolismo celular basal e do consumo de energia. De acordo com a equação de Arrehenius, a relação logarítmica das reações químicas é inversamente proporcional à temperatura absoluta. O múltiplo pelo qual as reações bioquímicas diminuem para cada $10^{\circ} \mathrm{C}$ é denominado Q10, sugerindo que o Q10 das crianças é de 3,65 comparado com aproximadamente 2,6 dos adultos. Valor que, maior nas crianças, sugere maior supressão metabólica relacionada à hipotermia, que permite tolerar períodos mais prolongados de isquemia. Durante a circulação extracorpórea, assim que a temperatura reduz, o consumo de oxigênio se torna independente do fluxo de perfusão. Este conceito é a base que definiu os fluxos de perfusão mínimos, para atender os requerimentos metabólicos, proporcionalmente diminuídos com a temperatura (tab. I).

Tradicionalmente, as recomendações para o fluxo de perfusão em crianças são baseadas no índice da massa corpórea. A adequação da perfusão durante a circulação extracorpóreaé monitorada pelos índices indiretos ou globais de perfusão tecidual. No passado, a pressão arterial invasiva adequada e a ausência de acidose metabólica eram considerados como marcadores de boa perfusão. O débito urinário durante a circulação extracorpórea pode ser indicador da perfusão renal, mas não serve como preditor de sobrevida e de complicações, ou seja, um paciente urinando não significa, necessariamente, que esteja bem do ponto de vista hemodinâmico.

Infelizmente, o cálculo dos fluxos tem mostrado baixa correlação com o metabolismo global e regional, e a isquemia tecidual ocorre durante alguns períodos da circulação extracorpórea, especialmente na presença de certos defeitos anatômicos, como a presença de colaterais sistêmicopulmonares ou de interrupção de arco aórtico, que requer alterações nas estratégias de perfusão. No sentido de melhor estimar a oferta de oxigênio aos tecidos durante a cir-

\begin{tabular}{|ccc|}
\hline $\begin{array}{c}\text { Tabela I - Fluxos de perfusão mínimos de acordo com a temperatura e } \\
\text { com a taxa de consumo de oxigênio cerebral (jaggers) }\end{array}$ \\
\hline \multirow{2}{*}{ Temperatura $\left({ }^{\circ} \mathrm{C}\right)$} & $\mathrm{VO}_{2}$ cerebral \\
$(\mathrm{mL} / 100 \mathrm{~g} / \mathrm{min})$ & $\begin{array}{c}\text { Fluxos mínimos } \\
(\mathrm{mL} / \mathrm{kg} / \mathrm{min})\end{array}$ \\
37 & 1,48 & 100 \\
32 & 0,82 & 56 \\
30 & 0,65 & 44 \\
28 & 0,51 & 34 \\
25 & 0,36 & 24 \\
20 & 0,20 & 14 \\
18 & 0,15 & 11 \\
15 & 0,11 & 8 \\
\hline
\end{tabular}


culação extracorpórea pediátrica, muitos Centros de cirurgia cardíaca abandonaram o tradicional cálculo do fluxo baseado na superfície corpórea ou no peso. Esses Centros adotaram a saturação venosa mista de oxigênio $\left(\mathrm{SvO}_{2}\right)$ como índice de perfusão tecidual, e tem sido o método de eleição na estimativa do fluxo de perfusão.

Por outro lado, o valor preditivo da $\mathrm{SvO}_{2}$ para refletir adequada perfusão tecidual durante a circulação extracorpórea requer hematócrito estável e adequado, profundidade da anestesia e consumo de oxigênio. A diminuição da saturação venosa de $\mathrm{O}_{2}$ durante a circulação extracorpórea pode ocorrer mesmo com fluxos e pressões de perfusão adequados, refletindo uma elevada extração de $\mathrm{O}_{2}$ devida ao processo fisiológico de aquecimento ou aos períodos de superficialização da anestesia. Apesar de manter $\mathrm{SvO}_{2}$ em níveis normais durante a circulação extracorpórea, complicações pós-operatórias sérias, potencialmente relacionadas à isquemia regional não detectada, continuam a ser relatadas.

Postula-se ${ }^{24}$,que a $\mathrm{SvO}_{2}$ possa não representar com acurácia a hipoperfusão tecidual, porque é reflexo da soma de todo o sangue venoso proveniente de todos os órgãos. Aqueles que recebem maior porcentagem do débito cardíaco, como o rim e o músculo esquelético, são os que contribuem com a maior porcentagem do retorno venoso. Por outro lado, órgãos com menor débito cardíaco, como o cérebro e o trato gastrointestinal, contribuem com menor proporção no sangue venoso misto, podendo ser a $\mathrm{SvO}_{2}$ pouco sensível na detecção da isquemia desses órgãos, com implicações clínicas extremamente relevantes.

McDaniel e cols. ${ }^{24}$ mostraram que durante $2 \mathrm{~h}$ de circulação extracorpórea normotérmica, $\mathrm{a} \mathrm{SvO}_{2}$ não refletiu a presença e gravidade de isquemia cerebral e intestinal e demonstraram, ainda, que o fluxo de perfusão de $100 \mathrm{~mL} / \mathrm{Kg}$ / min durante a circulação extracorpórea normotérmica com hemodiluição não previniu a diminuição na $\mathrm{SvO}_{2}$. A desaturação venosa regional e a acidose metabólica podem ser causadas por redução do débito cardíaco durante a circulação extracorpórea, como nos períodos de baixo fluxo de perfusão. Porém, pode também ser secundária a redistribuição do fluxo sanguíneo entre os órgãos durante a circulação extracorpórea, levando a hipoperfusão de alguns tecidos mais vulneráveis e desaturação venosa, que, por sua vez, pode estar relacionada a distribuição heterogênea do fluxo sanguíneo num mesmo órgão, como por exemplo, o trato gastrointestinal. Finalmente, a resposta inflamatória sistêmica pode causar hipóxia tecidual e lesão irreversível, pela inabilidade de manter o consumo e atender ao maior requerimento energético do organismo.

Apesar de ser inespecífico, o aumento ou a mudança dos níveis de lactato durante a circulação extracorpórea, pode ser também considerado marcador de hipoperfusão regional ou de aumento da demanda metabólica. Os órgãos que mais frequentemente produzem lactato em resposta à hipoperfusão ou à diminuição na extração de $\mathrm{O}_{2}$ incluem o cérebro, intestinos, fígado, rins, e músculos esqueléticos ${ }^{25,26}$. A análise do lactato sérico, como métodopara estimar a perfusão tecidual durante a circulação extracorpórea e a sua correlação com $\mathrm{S} \mathrm{SvO}_{2}$ é motivo de estudo em andamento.
Mudanças no fluxo sanguíneo cerebral em resposta à parada circulatória total tem sido descritas ${ }^{27}$ e os métodos utilizados para sua monitorização incluem a mensuração da saturação de $\mathrm{O}_{2}$ no bulbo venoso jugular ${ }^{28}$ e a espectroscopia infra-vermelha. Esses índices não mostraram correlação com o lactato sérico.

Ecocardiografia - Está sempre disponível? E quando está, o examinador é experiente? - A ecocardiografia constituiu, nas últimas décadas, um dos maiores avanços da cardiologia pediátrica. O desenvolvimento tecnológico permitiu equipamentos e transdutores mais sensíveis e, inclusive, o uso de transdutores transesofágicos.

Inicialmente, seu papel na cirurgia cardíaca se restringia a avaliação transtorácica no pós-operatório, tendo importância fundamental no diagnóstico de alterações anatomofuncionais responsáveis por evolução conturbada, avaliação da função ventricular global e regional e/ou presença de derrames pericárdicos previamente à alta hospitalar.

As indicações da ecocardiografia são vastas e, a partir do final da década de 1980, seu emprego invadiu o centro cirúrgico, tornando-se atualmente a ecocardiografia transesofágica intra-operatória um método consagrado e rotineiro de avaliação hemodinâmica. Os benefícios são evidentes porque permite a confirmação preliminar do resultado cirúrgico, e o diagnóstico e a estimativa de lesões residuais que possam ser prontamente corrigidas ${ }^{29}$. Este fato foi avaliado por alguns trabalhos ${ }^{29-31}$ que estudaram o impacto da ecocardiografia transesofágica intra-operatória sobre lesões residuais logo após a saída de circulação extracorpórea. O diagnóstico dessas lesões resultou em período adicional de circulação extracorpórea em 5 a $11 \%$ dos pacientes. Além disso, a ecocardiografia transesofágica intra-operatória detectou lesões não diagnosticadas no pré-operatório em 3 a $39 \%$ dos $\operatorname{casos}^{31-33}$, levando a mudanças no planejamento cirúrgico. As anomalias congênitas que mais se beneficiaram do procedimento, em termos de impacto no tratamento cirúrgico, foram as obstruções complexas da via de saída de ventrículo direito, a transposição das grandes artérias, a dupla via de saída do ventrículo direito, as discordâncias atrioventriculares, a estenose subaórtica e o defeito parcial e total do septo atrioventricular ${ }^{31}$. Outros benefícios são a visibilização de ar nas cavidades cardíacas na saída de circulação extracorpórea, o auxílio para o posicionamento de cateteres intracavitários, além de estimar em tempo real a contratilidade miocárdica e a volemia até o paciente ser transferido para a UTI, permitindo maior segurança e informação para o intensivista.

O progresso tecnológico com a minituarização dos equipamentos levou à redução significativa das complicações do procedimento, ampliando seu emprego a crianças pequenas e neonatos. Entretanto, este último ainda é considerado o grupo mais susceptível a problemas, especialmente aqueles com menos de $5 \mathrm{Kg}$ de peso. De acordo com Stevenson ${ }^{34}$, a incidência global de complicações relacionadas ao procedimento em crianças é de $3,2 \%$ e incluem a falha na introdução do transdutor $(0,8 \%)$, obstrução de vias aéreas $^{35}(0,8 \%)$, deslocamento do tubo endotraqueal $(0,2 \%)$, 
extubação inadvertida $(0,5 \%)$, compressão vascular $(0,6 \%)$ e trauma da orofaringe, esôfago e laceração da mucosa bucal $(0,4 \%)$. O comprometimento hemodinâmico ${ }^{36}$, apesar de raro, exige suspeita clínica e conduta imediatas e adequada interação entre cirurgião e anestesista. Os mecanismos responsáveis por alterações circulatórias podem envolver a compressão da aorta descendente ${ }^{37}$, átrio esquerdo, veias pulmonares, veia cava superior, artéria pulmonar ou indução de arritmias.

Apesar de todas essas considerações positivas a respeito do amplo emprego da ecocardiografia transesofágica intra-operatória em cirurgia cardíaca pediátrica, algumas questões devem ser esclarecidas: 1) deve ser utilizada em todos os casos, mesmo nos mais simples como fechamento de comunicação interatrial? ${ }^{38}$ Qual seria seu custo-benefício? 2) os riscos se sobrepõem aos benefícios em neonatos? 3) melhora a sobrevida? 4) tem limitações diagnósticas?

Não há dúvida de que a ecocardiografia transesofágica intra-operatória é um instrumento diagnóstico valioso ${ }^{39}$. Os argumentos ao uso rotineiro do procedimento, focaliza custo e risco de complicações relacionadas ao procedimento, versus os benefícios ao paciente. Os índices de reexploração estão diretamente relacionados à experiência do grupo clínico-cirúrgico, e devem ser analisados com critério. Randolph e cols. ${ }^{31}$ provaram, que grupos com grande experiência no tratamento das cardiopatias congênitas, utilizando esquema seletivo de exames, não alteraram os resultados cirúrgicos. Resta saber se a tática pode ser adotada em grupos com menor experiência. Avaliando a realidade do nosso meio, a maioria dos centros de cirurgia cardíaca pediátrica não dispõem de ecocardiografia intra-operatória confiável, por falta de equipamentos adequados e pela limitação de pessoal treinado.

Em relação à qualificação profissional para realização dos exames, não existem recomendações globais, sendo inclusive realizada, em alguns centros, por médicos anestesiologistas ${ }^{40}$. Devido à complexidade do exame, certas normas foram estabelecidas em relação a sistematização da avaliação, devendo ser mais criteriosa que a realizada em pacientes com cardiopatias adquiridas ${ }^{41}$.

Alguns autores ${ }^{42,43}$ demostraram que existe uma relação favorável de custo-benefício na realização rotineira da ecocardiografia transesofágica intra-operatória durante a cirurgia cardíaca pediátrica. $\mathrm{O}$ custo médio dos pacientes que necessitaram de reoperação por defeitos residuais, foi maior naqueles que retornaram da UTI após uma permanência variável, em comparação com aqueles reoperados durante o mesmo ato cirúrgico.

Apesar de todos os benefícios do procedimento deve-se lembrar, que o uso de cateteres intracavitários tem valor inestimável no diagnóstico de lesões residuais, alertando o cirurgião em relação a shunts residuais e obstruções da via de saída dos ventrículos, mais prontamente que a ecocardiografia transesofágica intra-operatória. Muitos desses cateteres permanecem no pós-operatório, podendo as medidas serem analisadas continuamente em tempo real. A ecocardiografia transesofágica intra-operatória apresen-
Monitorização hemodinâmica em cirurgia cardíaca pediátrica

ta limitações técnicas, principalmente devido à influência do estado hemodinâmico comumente presente logo após a saída de circulação extra-corpórea, onde volemia e função ventricular estão longe dos ideais. Desta forma, a estimativa de disfunções valvares e estenose subaórtica são alguns dos exemplos de situações nas quais o procedimento traz freqüentemente interpretações conflitantes. A avaliação da função ventricular impõe grande limitação ao método, na medida que requer inferências anatômicas da morfologia dos ventrículos, estando sujeita a variabilidade de acordo com o examinador. A recuperação do miocárdio após período de circulação extracorpórea, o uso de drogas inotrópicas, a presença de distúrbios do ritmo cardíaco e a volemia, são fatores adicionais a serem considerados. Por estes motivos alguns autore ${ }^{39}$ consideram, que os marcadores bioquímicos teriam maior validade na estimativa do débito cardíaco que a ecocardiografia.

Assim sendo, a influência dos achados ecocardiográficos na determinação e conduta terapêuticas deve ser feita, levando em consideração o estado hemodinâmico e, também, as limitações anatômicas e cirúrgicas de cada paciente. A análise é dependente do examinador, que obviamente deve estar capacitado, exigindo experiência e completo entendimento da anatomia e fisiopatologia das cardiopatias congênitas, bem como do procedimento cirúrgico em questão. Cabe ao cirurgião saber discernir os achados ecocardiográficos dentro do contexto clínico e dos achados intraoperatórios. A decisão de retornar à circulação extracorpórea para a correção de defeitos residuais, muitas vezes torna-se penosa, exigindo experiência e coragem, pesando sempre os riscos e benefícios de um período adicional de anóxia miocárdica.

Gasometria - As informações obtidas são valorizadas? - A gasometria arterial é um dos exames mais realizados na prática médica, especialmente nas unidades de terapia intensiva. Constitui exame de rotina no pós-operatório imediato de cirurgia cardíaca pediátrica, como método de adequação da ventilação mecânica e de seu desmame, e no diagnóstico de distúrbios do equilíbrio ácido-básico. Sua determinação seriada é fundamental na confirmação da eficácia das medidas terapêuticas eventualmente instituidas. Nenhum dos parâmetros da gasometria arterial medidos isoladamente é considerado método de monitorização hemodinâmica. Entretanto, estes parâmetros combinados podem estimar indiretamente a oferta de $\mathrm{O}_{2}$ e a perfusão tecidual ${ }^{44}$.

Além dos parâmetros vastamente conhecidos, algumas variáveis podem ser determinadas baseadas nos valores dos gases arteriais e venosos (fig. 2). Assim, tem importância na estimativa do quadro hemodinâmico, o cálculo dos conteúdos arterial e venoso de $\mathrm{O}_{2}$ e a diferença da saturação arteriovenosa.

No pós-operatório de cirurgia cardíaca pediátrica, ocorre aumento do consumo de oxigênio nas primeiras horas, diretamente relacionado a mudanças da temperatura central ${ }^{45}$. Este aumento do $\mathrm{VO}_{2}$ é acompanhado pelo $\mathrm{DO}_{2}$, e nesse período não ocorrem alterações expressivas da extração de $\mathrm{O}_{2}^{45}$. 


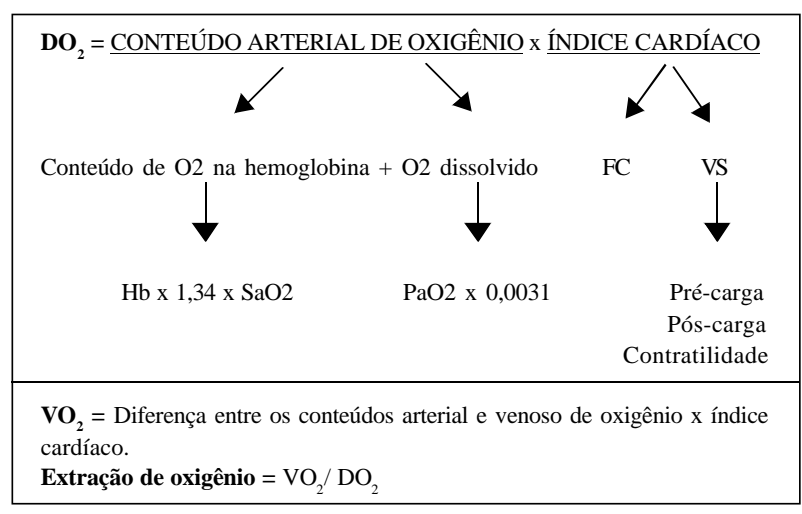

Fig. 2 - Determinantes da oferta de oxigênio $\left(\mathrm{DO}_{2}\right)$, do consumo de oxigênio $\left(\mathrm{VO}_{2}\right)$ e da extração de oxigênio. FC: frequência cardíaca; VS: volume sistólico; Hb: hemoglobina; $\mathrm{SaO}_{2}$ : saturação arterial de oxigênio; $\mathrm{PaO}_{2}$ : pressão parcial de oxigênio.

A extração de $\mathrm{O}_{2}$ pode refletir melhor o balanço do transporte de $\mathrm{O}_{2}$, particularmente após cirurgia cardíaca pediátrica, onde a oferta de $\mathrm{O}_{2}$ pode estar limitada pela hipoxemia. Foi sugerido que quando a extração de $\mathrm{O}_{2}$ se encontra entre 0,5 e 0,6 , o consumo se torna patologicamente dependente da oferta de $\mathrm{O}_{2}$, ponto considerado crítico que está diretamente associado à acidose láctica. Uma grande vantagem da extração de $\mathrm{O}_{2}$ reside na facilidade da sua determinação, através de exames rotineiros no pós-operatório. Rossi e cols. ${ }^{46}$, estudando seu valor prognóstico, encontraram que a extração de pelo menos 0,5 foi fator de risco de mortalidade, presente em $70 \%$ dos não sobreviventes em comparação com $25 \%$ dos sobreviventes.

O cálculo do débito cardíaco é obtido pelo método da termodiluição ou utilizando o princípio de Fick. O primeiro exige um cateter de Swan-Ganz e monitores especiais, os quais se tornam inviáveis na população pediátrica, enquanto que a outra opção requer um método confiável de captação do oxigênio nos pulmões. Ambos os métodos sofrem influências de shunts intracardíacos ${ }^{47}$. Considerando as limitações de ambos os métodos, vários autores sugerem que o débito cardíaco pode ser estimado pela saturação venosa mista de $\mathrm{O}_{2}\left(\mathrm{SvO}_{2}\right)$.

$\mathrm{A} \mathrm{SvO}_{2}$ é geralmente aceita como indicador da adequação do débito cardíaco e apresenta elevada associação com acidose láctica em terapia intensiva ${ }^{48}$. Considerando o conteúdo arterial de $\mathrm{O}_{2}$ e o metabolismo celular estáveis e adequados, a equação de Fick determina que $\mathrm{SvO}_{2}$ maior que $60 \%$, é compatível com débito cardíaco adequado. A vantagem da $\mathrm{SvO}_{2}$ está na capacidade de estimar tanto a oferta quanto o consumo de $\mathrm{O}_{2}$. A diminuição da $\mathrm{SvO}_{2}$ é dependente não somente da redução do índice cardíaco, mas também da hemoglobina, da oxigenação arterial, e/ou do maior consumo de $\mathrm{O}_{2}$ dos tecidos ${ }^{49}$. Todos esses fatores desempenham papel importante na manutenção do choque. Este é um fator limitante do uso da $\mathrm{SvO} 2$ em cirurgia cardíaca pediátrica, pela freqüente presença de hipoxemia em crianças cianóticas, que certamente afeta sua sensibilidade e especificidade. O mesmo ocorre com a diferença arteriovenosa de $\mathrm{O}_{2}$, outro parâmetro utilizado na estimativa da perfusão tecidual ${ }^{47}$.
Mas o que é $\mathrm{SSvO}_{2}$ e qual é o local ideal de ser colhida? $\mathrm{A} \mathrm{SvO}_{2}$ é a mistura de todo o retorno venoso sistêmico, e a artéria pulmonar é o local mais adequado para sua mensuração. Entretanto, em cardiologia pediátrica, a artéria pulmonar é um péssimo local, pois qualquer shunt da esquerda para a direita causará falsamente elevação da saturação. Alguns autores ${ }^{50,51}$ validaram a coleta de sangue do átrio direito e veia cava superior como estimativas confiáveis da $\mathrm{SvO}_{2}$. Tweddell e cols. ${ }^{52}$ desenvolveram um método de monitorização oximétrica continua da $\mathrm{SvO}_{2}$ na veia cava superior. A estimativa do débito cardíaco e da relação ideal de fluxos pulmonar e sistêmico ${ }^{53}$, são as informações colhidas a partir dessa análise, sendo considerada o método de escolha no pós-operatório da operação de Norwood.

\section{Marcadores bioquímicos}

Lactato-O valor normal do lactato séricoé de $1 \mathrm{mmol} / \mathrm{L}$, estendendo-se até $2 \mathrm{mmol} / \mathrm{L}$ em pacientes críticos. A elevação desses índices pode ocorrer com ou sem acidose metabólica concomitante, e a distinção é muito importante. A hiperlactatemia é considerada de grau leve a moderado ( 2 a $5 \mathrm{mmol} / \mathrm{L})$ quando não há acidose metabólica, enquanto que na acidose láctica há elevação acentuada e persistente do lactato sérico (habitualmente $>5 \mathrm{mmol} / \mathrm{L}$ ) associado à acidose metabólica.

A hiperlactatemia ${ }^{54}$ geralmente ocorre em situações nas quais os tecidos são bem perfundidos, tendo como mecanismos desencadeadores, fatores que aumentam a glicólise anaeróbica, como o uso de catecolaminas. Nesse caso, os sistemas tampões permanecem intactos, o que mantém o pHem níveis normais.

Já a acidose láctica resulta da hipoperfusão tecidual, do efeito de certas drogas, ou de defeitos inatos do metabolismo dos carboidratos. A oferta de $\mathrm{O}_{2}$ é determinada pelo débito cardíaco e pelo conteúdo arterial de $\mathrm{O}_{2}$, sendo as maiores causas de acidose láctica aquelas associadas ao comprometimento cardiopulmonar, como o choque e a insuficiência respiratória aguda. A anemia e a hipoxemia diminuem o conteúdo arterial de $\mathrm{O}_{2}$, mas a sua oferta aos tecidos tende a ser mantida por mecanismos compensatórios que elevam o débito cardíaco. Quando ocorre acidose láctica associada a esses fatores, provavelmente existe comprometimento cardiovascular concomitante.

Durante condições de metabolismo anaeróbico, o piruvato (produto final da glicólise) é convertido em lactato pela transferência de dois átomos de hidrogênio da coenzima ADP. Essas alterações no metabolismo do lactato são freqüentemente encontradas em pacientes críticos que apresentam maior risco de falência de múltiplos órgãos e óbito. Vários estudos têm mostrado forte associação entre a elevação do lactato sérico e maior morbidade e mortalidade. Esses estudos examinaram várias populações, incluindo choque séptico ${ }^{55}$, infarto do miocárdio, pós-operatório de cirurgias abdominais, trauma e insuficiência respiratória do recém-nascido ${ }^{56}$.

Em cirurgia cardíaca, vários estudos têm demonstrado a eficácia do lactato sérico como preditor de mortalidade em 
pacientes adultos ${ }^{57}$. A elevação do lactato sérico à admissão na terapia intensiva foi proposta como um marcador potencial de má evolução no pós-operatório de cirurgia cardíaca pediátrica. Nessa população, a elevação do lactato deve ser motivo de investigação das condições que levaram à hipoperfusão tecidual. De maneira geral, incluem a adaptação do aparelho cardiovascular e respiratório às mudanças da volemia no pós-operatório, aos efeitos da circulação extracorpórea sobre o organismo e à presença de defeitos residuais.

Entretanto, os diversos estudos que analisaram seu valor prognóstico, não definiram um valor específico de sobrevida. Hatherill e cols. ${ }^{58}$ relataram que, o valor de lactato sérico $>6 \mathrm{mmol} / \mathrm{L}$ à admissão, tem baixo valor preditivo de mortalidade em cirurgia cardíaca pediátrica, considerada injustificada a mensuração rotineira. Cheifetz e cols. ${ }^{59}$ encontraram que o lactato inicial $>7 \mathrm{mmol} / \mathrm{L}$ e máximo acima de $9 \mathrm{mmol} / \mathrm{L}$ durante a internação, são preditores de mortalidade. Outros autores ${ }^{60}$ confirmaram os mesmos achados, porém com valores séricos menores. Já Charpie e cols. ${ }^{61}$ encontraram que o lactato inicial maior que $6 \mathrm{mmol} / \mathrm{L}$ tem valor preditivo baixo (38\%) para má evolução na população neonatal, dando maior importância para valores acima de 0,75 por hora, com elevada associação com óbito, conceito que é extremamente importante, dando-se maior relevância à mensuração seriada e podendo servir como guia da resposta terapêutica e evolutiva em alguns $\operatorname{casos}^{62}$. Conclui-se pelas evidências da literatura ${ }^{59,61}$, que o lactato é um bom preditor de mortalidade em cirurgia cardíaca pediátrica e que, além de sua elevação, a mensuração seriada é importante na determinação da tendência, sendo um potente indicador de óbito, o que justifica a realização do diagnóstico mais acurado e da terapêutica mais agressiva. As elevações discretas do lactato ( 2 a $4 \mathrm{mmol} / \mathrm{L})$, freqüentemente encontradas no pós-operatório, devem ser analisadas com cautela, já que não refletem o desequilíbrio entre oferta e consumo de $\mathrm{O} 2$, não servindo portanto como marcadores de sobrevida ${ }^{45}$.

Além disso, o momento ideal de sua monitorizaçãoé tema que deve ser discutido. Munoz e cols.$^{62}$ provaram que a elevação do lactato durante a circulação extracorpórea pode ser um marcador mais precoce de sobrevida que a monitorização realizada a partir do pós-operatório, postulando que os processos fisiopatológicos se iniciam durante o ato operatório. Desta forma, medidas terapêuticas poderiam ser adotadas mais precocemente, com provável profilaxia de eventos catastróficos no pós-operatório. Houve relação positiva entre a hiperlactatemia e os óbitos na cirurgia de Norwood em neonatos, maior tempo de circulação extracorpórea e parada circulatória total, maior pontuação no PRISM III à admissão em UTI, maior tempo de ventilação mecânica e de permanência em UTI.

Rossi e cols. ${ }^{46}$ destacaram algumas desvantagens do lactato como método de monitorização hemodinâmica, postulando que a elevação seja tardia, numa fase em que o consumo é dependente da oferta. Além disso, a elevação no pós-operatório pode representar instabilidade hemodinâmica prévia, intra ou até pré-operatória em situações de metabolização hepática comprometida.
Monitorização hemodinâmica em cirurgia cardíaca pediátrica

Troponina - A troponina é um marcador bioquímico de lesão miocárdica durante a cirurgia cardíaca. A troponina é um componente do sistema contrátil da musculatura estriada que, conjuntamente com a tropomiosina, aumenta a sensibilidade à entrada de cálcio na formação de complexos de actina e miosina, responsável pela contração miocárdica. A troponina está presente sob duas formas, troponina $\mathrm{T}$ e troponina I, ambas miocárdio específicas, embora a primeira apresente baixo valor preditivo negativo em pacientes com deficiência da função renal ${ }^{63}$. A troponina T é uma proteína com peso molecular de 39,7 kD com alta sensibilidade para lesão miocárdica. A sua determinação bioquímica é considerada como método estabelecido de lesão miocárdica em pacientes adultos após o infarto agudo do miocárdio, tendo elevações séricas por volta de 3 a 4 h após a ocorrência de sintomas e pode permanecer alterada por até 14 dias.

Na população pediátrica, Hirsch e cols. ${ }^{64}$ encontraram que a troponina I não está alterada no pré-operatório das cardiopatias congênitas ou na população pediátrica em geral e mostraram que os valores de troponina I estão usualmente abaixo de $2 \mathrm{ng} / \mathrm{ml}$, considerados assim como normais.

No pós-operatório de cirurgia cardíaca pediátrica, Taggart e cols. ${ }^{65}$ mostraram que a troponina é um marcador de lesão miocárdica mais sensível que a fração miocárdica da creatinoquinase e que a mioglobina. Considerando essa população, a lesão miocárdica ${ }^{66}$ pode ocorrer durante infarto ou trauma sobre o músculo cardíaco, tanto como resultado de incisão cirúrgica direta, quanto por problemas na circulação coronariana (por exemplo a transferência inadequada dos óstios coronarianos na operação de Jatene) ou ainda, secundária à proteção miocárdica inadequada. Variações nas técnicas operatórias e diferenças nas respostas dos pacientes aos métodos de preservação miocárdica também estão envolvidas nesses mecanismos de lesão, pela vulnerabilidade variável do miocárdio à hipóxia ${ }^{67}$.

Pelo fato da troponina refletir a intensidade da lesão miocárdica no período perioperatório, alguns autores ${ }^{66,68}$ têm sugerido que ela possa ser fator prognóstico no pós-operatório de cirurgia cardíaca pediátrica, podendo estimar a intensidade e a duração das complicações relacionadas a um tipo de operação em específico ou tipo de tratamento clínico. Entretanto, esta hipótese deve ser confirmada em estudos prospectivos.

\section{Tonometria gástrica}

O trato gastrointestinal é um dos primeiros órgãos a serem afetados em situações de hipoperfusão tecidual, devido à sua relativa vulnerabilidade à hipóxia, explicada pela estrutura vascular de arteríolas e vênulas que correm em paralelo, permitindo uma depleção de $\mathrm{O}_{2}$ e acúmulo de gás carbônico $\left(\mathrm{CO}_{2}\right)$ nos tecidos.

Ocorre ainda aumento da produção de dióxido de carbono pela hidrólise do ATP, com grande acúmulo de íons hidrogênio na célula, de acordo com a equação de Henderson-Hasselbach, fenômeno bioquímico que tem importância fundamental, já que a detecção de alterações na concen- 
tração de $\mathrm{CO}_{2}$ tecidual e do $\mathrm{pH}$ intramucoso, podem servir de marcadores de hipoperfusão em terapia intensiva, antes que ocorram alterações clínicas da pressão arterial, da acidose metabólica, da pressão arterial sistêmica e da frequência cardíaca ${ }^{69}$. Estudos experimentais demonstraram associação positiva entre o $\mathrm{pH}$ da mucosa do trato gastrointestinal e a perfusão tecidual ${ }^{70}$, reafirmando que o $\mathrm{pH}$ intramucoso é um marcador sensível do metabolismo anaeróbico e da diminuição na oferta de $\mathrm{O}_{2}^{71-73}$

A tonometria foi desenvolvida em 1959 como um método de mensuração da pressão parcial do $\mathrm{CO}_{2}$ em crianças portadoras de poliomielite, submetidas à ventilação mecâni$\mathrm{ca}^{74}$. Desde seu primeiro uso clínico, diversas modificações foram feitas, sendo atualmente a tonometria um método relativamente não invasivo, que permite a mensuração do $\mathrm{pH}$ intramucoso ou do $\mathrm{CO}_{2}$ tecidual do trato gastrointestinal. Consiste em um balão de silicone acoplado a uma sonda digestiva que tem permeabilidade ao $\mathrm{CO}_{2}$. Seu princípio baseia-se, na elevada capacidade de difusão do $\mathrm{CO}_{2}$ pelos tecidos e concentração constante de bicarbonato entre a mucosa intestinal e o sangue arterial ${ }^{75}$. A tonometria pode ser empregada em vários locais do trato gastrointestinal. $\mathrm{O}$ mais freqüente é o gástrico, pela disponibilidade de ser acoplada a uma sonda que teria a função adicional de descompressão gástrica. Pode ainda ser utilizada em outros locais, como o cólon sigmóide, intestino delgado ${ }^{76}$ e esôfago ${ }^{77}$.

A aplicação clínica da tonometria ocorreu inicialmente na população adulta e vários estudos mostraram seu valor preditor de morbidade e mortalidade numa variedade de condições clínicas e cirúrgicas ${ }^{78-81}$, inclusive em cirurgia cardíaca $^{82}$. Baseados nos estudos de Gys e cols. ${ }^{83}$, que definiram o pH intramucoso abaixo de 7,32 a 7,35 como fator independente de risco de mortalidade em adultos a técnica, foi incorporada como método de monitorização hemodinâmica em alguns centros de terapia intensiva.

Na população pediátrica, a determinação do $\mathrm{pH}$ da mucosa gástrica pela tonometria provou ser um método fidedigno na mensuraçãoção da perfusão esplâncnica ${ }^{84}$. Entretanto, em cirurgia cardíaca pediátrica, seu valor como preditor de morbidade e mortalidade é controverso ${ }^{85,86}$. Pérez e cols. ${ }^{87}$ encontraram associação entre o $\mathrm{pH}$ intramucoso $\leq 7,32$ à admissão e $12 \mathrm{~h}$ após e a presença de insuficiência de múltiplos órgãos e sistemas com maior mortalidade no pós-operatório de cirurgia cardíaca pediátrica. Soybir e cols. ${ }^{88}$ estudando pacientes submetidos a correção da coarctação de aorta, encontraram alterações do $\mathrm{CO}_{2}$ gástrico durante o pinçamento da aorta, com normalização posterior, sem nenhuma repercussão clínica. Souza e cols. ${ }^{89}$, em nosso meio, encontraram alterações temporárias do pH intramucoso no pós-operatório de cirurgia cardíaca pediátrica com circulação extracorpórea, sem associação com fatores mórbidos ou óbito. Baseados nos achados de seu estudo, questionam: por quanto tempo pode ocorrer acidose da mucosa gástrica até que apareçam alterações clínicas?

Apesar de promissora aplicação prática, a tonometria não foi incorporada amplamente à prática médica, por uma série de motivos: 1) a isquemia da mucosa do trato gastroin- testinal pode refletir a hipoperfusão sistêmica? 2) a tonometria é um método confiável como guia terapêutico?3) Existe valor prognóstico e comportamento normal? O que significa quando está alterada?

As limitações da tonometria gástrica não são desprezíveis, conforme alguns estudos ${ }^{76}$. O refluxo do suco duodenal alcalino e a retrodifusão do bicarbonato da mucosa gástrica, produzem uma quantidade significativa de $\mathrm{CO}_{2}$ não relacionada à hipoperfusão tecidual. Além disso, o equilíbrio ácido-básico da mucosa gástrica é complexo e a relação entre o $\mathrm{pH}$ intramucoso e a perfusão tecidual pode não ser precisa $^{90}$. Os tonômetros requerem um período de equilíbrio, muitas vezes demorado, exigindo que o cálculo considere um fator de correção, o que pode levar a erros ${ }^{91}$.

Quando comparada com outros métodos de perfusão, seus resultados são controversos. Apesar de seus valores serem considerados significativos em grupos selecionados, o seu uso como guia individual não se provou eficaz ${ }^{90}$. Desta forma, até que o custo/benefício e a análise desses fatores indiquem o contrário, o uso rotineiro em cirurgia cardíaca pediátrica não é recomendado.

Cateterismo cardíaco - O progresso alcançado no campo da ecocardiografia em termos diagnósticos eliminou muitas indicações do estudo hemodinâmico em cardiologia pediátrica. Por outro lado, o cateterismo cardíaco, atualmente, desenvolve-se exponencialmente no campo intervencionista $^{92}$, sendo parceiro fiel da cirurgia na minimização dos riscos e obtenção de resultados mais adequados. A integração dos métodos inicia-se no período pré-operatório com melhoria das condições clínicas, como por exemplo, na estenose pulmonar valvar crítica, tratada com cateter balão, ou na transposição das grandes artérias com septo interventricular intacto, utilizando-se a atriosseptostomia, entre outras.

Tradicionalmente, pacientes com distúrbios cardiocirculatórios e pulmonares no pós-operatório, que não são resolvidos pelas medidas usuais, devem obrigatoriamente ser investigados em relação à presença de defeitos residuais. A suspeita inicial deve ser confirmada, preferencialmente, pela ecocardiografia. O cateterismo cardíaco estará indicado em condições de dúvidas do resultado ecocardiográfico, às vantagens da aferição direta da manometria das cavidades cardíacas, das medidas de fluxos e de resistências vasculares sistêmica e pulmonar, à maior precisão no cálculo de gradientes das vias de saída dos ventrículos, auxiliando assim no planejamento da nova intervenção cirúrgica. No período pós-operatório, a grande vantagem do método está no seu caráter intervencionista, que além de confirmar inicialmente a suspeita ecocardiográfica, pode resolver o problema residual sem a necessidade de nova cirurgia. Isso ocorre por exemplo na embolização de vasos colaterais sistêmico-pulmonares da atresia pulmonar com comunicação interventricular, entre outros.

\section{Conclusões}

Os progressos alcançados no tratamento das cardiopatias congênitas foram fantásticos na última década, e os 
resultados são fruto de uma série de fatores, mas sem dúvida, desempenhando papel fundamental, a monitorização hemodinâmica.

Oentendimento da fisiopatologia das cardiopatias congênitas, aliado ao conhecimento das relações entre oferta e consumo de oxigênio ao nível celular são a chave do sucesso no manejo intra e pós-operatório em cirurgia cardíaca pediátrica.

Não se deve esquecer que nenhum método é infalível na detecção da hipoperfusão tecidual. A monitorização hemodinâmica deve ser iniciada no período pré-operatório, ser a mais completa possível durante o ato operatório, e se manter no pós-operatório, até que não haja dúvidas em relação à
Monitorização hemodinâmica em cirurgia cardíaca pediátrica

evolução clínica. Todo tipo de monitorização depende da interpretação desempenhada pelo cérebro humano, cuja engenhosa máquina nunca foi ultrapassada pela tecnologia. A suspeita clínica, por mais sutil que seja, deve ser valorizada e investigada prontamente, na detecção de alterações que possam ser corrigidas a tempo. É de fundamental importância que haja uma harmonia profunda entre cardiologista pediátrico, cirurgião, anestesista, intensivista, hemodinamicista, ecocardiografista, enfermeiro, fisioterapeuta e nutricionista. A falha na estruturação e, principalmente, na manutenção deste conjunto, leva esta união, inexoravelmente, a erros, ou ainda o pior, ao óbito.

\section{Referências}

1. Azakie T, Merklinger SL, McCrindle BW, et al - Evolving strategies and improving outcomes of the modified norwood procedure: a 10-year single-institution experience. Ann Thorac Surg 2001; 72: 1349-53.

2. American Academy of Pediatrics, Section on Cardiology and Cardiac Surgery Guidelines for Pediatric Cardiovascular Centers. Pediatrics 2002; 109: 544-9.

3. Wo CCJ, Shoemaker WC, Appel PL, et al-Unrealiability of blood pressure and heart rate to evaluate cardiac output in emergency resuscitation and critical illness. Crit Care Med 1993; 21: 218-23.

4. Vincent JL - End-points of resuscitation: arterial blood pressure, oxygen delivery, blood lactate, or ...? Intensive Care Med 1996; 22: 3-5.

5. Duke T, Butt W, South M, et al -Early markers of major adverse events in children after cardiac operations. J Thorac Cardiovasc Surg 1997; 114: 1042-52.

6. Auler Jr JOC, Barreto AC, Gimenez SC, et al-Pediatric cardiac postoperative care. Rev Hosp Clin Fac Med S Paulo 2002; 57: 115-23.

7. Sano S, Brawn WJ, Mee RBB. Total anomalous pulmonary venous drainage. J Thorac Cardiovasc Surg 1989; 97: 886-92.

8. Pollack MM, Patel KM, Ruttimann UE-PRISM III: an updated pediatric risk of mortality score. Crit Care Med 1996; 24: 743-52.

9. Seghaye MC, Engelhardt W, Grabitz RG, et al. - Multiple system organ failure after open heart surgery in infants and children. Thorac Cardiovasc Surg 1993; 41: 49-53.

10. Proulx F, Gauthier M, Nadeau D, et al - Timing and predictors of death in pediatric patients with multiple organ system failure. Crit Care Med 1994; 22: 1025-31.

11. Shime N, Kageyama K, Ashida H, et al-Application of modified sequential organ failure assessment score in children after cardiac surgery. J Cardiothorac Vasc Anesth 2001; 15: 463-8.

12. Petros AJ, Marshall JC, van Saene HKF - Should morbidity replace mortality as an endpoint for clinical trials in intensive care? Lancet 1995; 345: 369-71.

13. Jaggers J, Shearer IR, Ungerleider RM - Cardiopulmonary bypass in infants and children. In: Gravlee GP, Davis RF, Kurusz M, Utley JR, eds. Cardiopulmonary bypass: principles and practice. Philadelphia, PA: Lippincott Williams \& Wilkins, 2000: 631-61

14. Lewis RC, Dural AM, Iliff A - Standards for basal metabolism in normal infants. J Pediatr 1943; 23: 1-18.

15. Benzing G III, Francis PD, Kaplan S, et al - Glucose and insulin changes in infants and children undergoing hypothermic open-heart surgery. Am J Cardiol 1983; 52: 133-6.

16. Shoemaker WC - Oxygen transport and oxygen metabolism in shock and critical illness. Invasive and noninvasive monitoring of circulatory dysfunction and shock. Crit Care Clin 1996; 12: 939-69.

17. Lugo G, Arizpe D, Dominguez G, et al - Relationship between oxygen consumption and oxygen delivery during anesthesia in high risk surgical patients. Crit Care Med 1993; 21: 64-9.

18. Anand KJS, Phil D, Hansen DD, et al - Hormonal-metabolic stress response in neonates undergoing cardiac surgery. Anesthesiology 1990; 73: 661-70.

19. Anand KJS, Phil D, Hickey PR - Halothane-morphine compared with high-dose sufentanil for anesthesia and postoperative analgesia in neonatal cardiac surgery. N Engl J Med 1992; 326: 1-9.

20. Lazenby WD, Ko W, Zelano JA, at al. - Effects of temperature and flow rate on regional blood flow and metabolism during cardiopulmonary bypass. Ann Thorac Surg 1992: 53: 957-64.
21. Rocke DA, Gaffin SL, Wells MT, et al - Endotoxemia associated with cardiopulmonary bypass. J Thorac Cardiovasc Surg 1987; 93: 832-7.

22. Smith EEJ, Naftel DC, Blackstone EH, et al - Microvascular permeability after cardiopulmonary bypass. J Thorac Cardiovasc Surg 1987; 94: 225-33.

23. Corno AF- What are the best temperature, flow, and hematocrit levels for pediatric cardiopulmonary bypass? J Thorac Cardiovasc Surg 2002; 124: 856-7.

24. McDaniel LB, Zwischenberger JB, Vertrees RA, et al - Mixed venous oxygen saturation during cardiopulmonary bypass poorly predicts regional venous saturation. Anesth Analg 1995; 80: 466-72.

25. Haisjackl M, Birnbaum J, Redlin M, et al. - Splanchnic oxygen transport and lactate metabolism during normothermic cardiopulmonary bypass in humans. Anesth Analg 1998; 86: 22-7.

26. Sicsic JC, Duranteau J, Corbineau H, et al. - Gastric mucosal oxygen delivery decreases during cardiopulmonary bypass despite constant systemic oxygen delivery. Anesth Analg 1998; 86: 455-60.

27. Jonas RA - Neurological protection during cardiopulmonary bypass/deep hypothermia. Pediatr Cardiol 1998; 19: 321-30.

28. Croughwell ND, White WD, Smith LR, et al. - Jugular bulb saturation and mixed venous saturation during cardiopulmonary bypass. J Card Surg 1995; 10: 503-8.

29. Rosenfeld HM, Gentles TL, Wernovcky G, et al - Utility of transesophageal echocardiography in the assessment of residual cardiac defects. Pediatr Cardiol 1998; 19: 346-51.

30. Stevenson JG, Sorenson GK, Hartman D, et al - Transesophageal echocardiography during repair of congenital heart defects: identification of residual problems necessitating reoperation. J Am Soc Echocardiog 1993; 6: 356-65.

31. Randolph GR, Hagler DJ, Connolly HM, et al. - Intraoperative transesophageal echocardiography during surgery for congenital heart defects. J Thorac Cardiovasc Surg 2002; 124: 1176-82.

32. Russell IAM, Miller-Hance WC, Silverman NH - Intraoperative transesophageal echocardiography for pediatric patients with congenital heart disease. Anesth Analg 1998; 87: 1058-76.

33. Ungerleider RM, Groeley WJ, Sheikh KH, et al - Routine use of intraoperative epicardial echocardiography and Doppler color-flow imaging to guide and evaluate repair of congenital heart lesions: a prospective study. J Thorac Cardiovasc Surg 1990; 100: 297-309.

34. Stevenson JG - Incidence of complications in pediatric transesophageal echocardiography. Experience in 1,650 cases. J Am Soc Echocardiog 1999; 12: 527-32.

35. Gilbert TB, Pancino FG, McGill WA, et al - Bronchial obstruction by transesophageal echocardiography probe in a pediatric cardiac patient. Anesth Analg 1992; 74: 156-8.

36. Andropoulos DB, Stayer SA, Bent ST, et al - The effects of transesophageal echocardiography on hemodynamic variables in small infants undergoing cardiac surgery. J Cardiothorac Vasc Anesth 2000; 14: 133-5.

37. Lunn RJ, Oliver WC, Hagler DJ, et al - Aortic compression by transesophageal echocardiography probe in infants and children undergoing cardiac surgery. Anesthesiology 1992; 77: 587-90.

38. Ramamoorthy C, Lynn AM, Stevenson JG - Pro: transesophageal echocardiography should be routinely used during pediatric open cardiac surgery. J Cardiothorac Vasc Anesth 1999; 13: 629-31.

39. McGowan FX Jr, Laussen PC - Con:transesophageal echocardiography should not be used routinely for pediatric open cardiac surgery. J Cardiothorac Vasc Anesth 1999; 13: 632-4. 
40. Pearlman A, Gardin J, Martin R, et al - Guidelines for physician training in transesophageal echocardiography: Recommendations of the American Society of Echocardiography Committee for Physician Training in Echocardiography. J Am Soc Echocardiog 1992; 5: 187-94.

41. Fyfe DA, Ritter SB, Snider AR, et al - Guidelines for transesophageal echocardiography in children. J Am Soc Echocardiog 1992; 5: 640-4.

42. Ungerleider RM, Kisslo IA, Greeley WJ, et al - Intraoperative echocardiography during congenital heart operations: experience from 1000 cases. Ann Thorac Surg 1995; 60(Suppl 6): S539-42.

43. Stevenson JG - Role of intraoperative transesophageal echocardiography during repair of congenital cardiac defects. Acta Pediatr Suppl 1995; 410: 23-33.

44. Zimmerman JL, Dellinger RP - Blood gas monitoring. Crit Care Clin 1996; 12: 865-74.

45. Li J, Schulze-Neick I, Lincoln C, et al. - Oxygen consumption after cardiopulmonary bypass surgery in children: determinants and implications. J Thorac Cardiovasc Surg 2000; 119: 525-33.

46. Rossi AF, Seiden HS, Gross RP, et al - Oxygen transport in critically ill infants after congenital heart operations. Ann Thorac Surg 1999; 67: 739-44.

47. Buheitel G, Scharf J, Hofbeck M, et al-Estimation of cardiac index by means of the arterial and the mixed venous oxygen content and pulmonary oxygen uptake determination in the early post-operative period following surgery of congenital heart disease. Intensive Care Med 1994; 20: 500-3.

48. Sumimoto T, Takayama Y, Iwasaka T, et al. - Mixed venous oxygen saturation as a guide to tissue oxygenation and prognosis in patients with acute myocardial infarction. Am Heart J 1991; 122: 27-33.

49. Kandel G, Aberman A - Mixed venous oxygen saturation. Arch Intern Med 1983; 143: 1400-2.

50. Thayssen $\mathrm{P}$, Klarholt E- Relation between caval and pulmonary artery oxygen saturation in children. Br Heart J 1980; 43: 574-8.

51. Tahvanainen J, Meretoja O, Nikki P-Can central venous blood replace mixed venous blood samples? Crit Care Med 1982; 10: 758-61.

52. Tweddell JS, Hoffman GM, Fedderly RT, et al. - Patients at risk for low systemic oxygen delivery after the Norwood procedure. Ann Thorac Surg 2000; 69: 1893-9.

53. Taeed R, Schwartz SM, Pearl JM, et al. - Unrecognized pulmonary venous desaturation early after Norwood palliation confounds Qp:Qs assessment and compromises oxygen delivery. Circulation 2001; 103: 2699-704.

54. Mizock BA - Lactic acidosis in critical illness. Crit Care Med 1992; 20: 80-93.

55. Bakker J, Coffernils M, Leon M, et al - Blood lactate levels are superior to oxygen-derived variables in predicting outcome in human septic shock. Chest 1991; 99: 956-62.

56. Cheung PY, Finer NN - Plasma lactate concentration as a predictor of death in neonates with severe hypoxemia requiring extra-corporeal membrane oxygenation. J Pediatr 1993; 125: 763-8.

57. Demers P, Elkouri S, Martineau R, et al-Outcome with high blood lactate levels during cardiopulmonary bypass in adult cardiac operation. Ann Thorac Surg 2000; 70: 2082-6

58. Hatherill M, Sajjanhar T, Tibby SM, et al.-Serum lactate as a predictor of mortality after pediatric cardiac surgery. Arch Dis Child 1997; 77: 235-8.

59. Cheifetz IM, Kern FH, Schulman SR, et al-Serum lactates correlate with mortality after operations for complex congenital heart disease. Ann Thorac Surg 1997; 64: 735-8.

60. Siegel LB, Dalton HJ, Hertzog JH, et al - Initial postoperative serum lactate levels predict survival in children after open heart surgery. Intensive Care Med 1996; 22: 1418-23.

61. Charpie JR, Dekeon MK, Goldberg CS, et al - Serial blood lactate levels during cardiopulmonary bypass for surgery for congenital heart disease: an early indicator of morbidity and mortality. J Thorac Cardiovasc Surg 2000; 120: 73-80.

62. Munoz R, Laussen PC, Palacio G, et al - Changes in whole blood lactate levels during cardiopulmonary bypass for surgery for congenital cardiac disease: an early indicator of morbidity and mortality. J Thorac Cardiovasc Surg 2000; 119: 155-62.

63. Immer FF, Stocker FP, Seiler AM, et al - Comparison of troponin-I and troponin-T after pediatric cardiovascular operation. Ann Thorac Surg 1998; 66: 2073-7.

64. Hirsch R, Landt Y, Porter S, et al - Cardiac troponin I in pediatrics: normal values and potential use in the assessment of cardiac injury. J Pediatr 1997; 130: 872-7.

65. Taggart DP, Hajinikolas L, Hooper J, et al. - Effects of age and ischemic times on biochemical evidence of myocardial injury after pediatric cardiac operations. J Thorac Cardiovasc Surg 1997; 113: 728-35.

66. Hirsch R, Dent CL, Wood MK, et al. - Paterns and potential value of cardiac tro- ponin I elevations after pediatric cardiac operations. Ann Thorac Surg 1998; 65: 1394-9.

67. Taggart DP, Hadjinikolas L, Wong K, et al. - Vulnerability of pediatric myocardium to cardiac surgery. Heart 1996; 76: 214-7.

68. Immer FF, Stoker F, Seiler AM, et al. - Troponin I for prediction of early postoperative course after pediatric cardiac surgery. J Am Coll Cardiol 1999; 33: 1719-23.

69. Hamilton-Davies C, Mythen M, Jacobson D, et al. - Comparison of commonly used clinical indicators of hypovolemia with tonometry. Crit Care Med 1995; 23(Suppl.): A86.

70. Grum CM, Fiddian-Green RG, Pittenger G, et al. - Adequacy of tissue oxygenation in intact dog intestine. J Appl Physiol 1984; 56: 1065-9.

71. Bass BL, Schweitzer BJ, Harmon JW, et al - Intraluminal p CO A reliable indicator of intestinal ischemia. J Surg Res 1985; 39: 351-60.

72. Hartman M, Montgomery A, Jonsson K, et al - Tissue oxygenation in hemorrhagic shock measured as transcutaneous oxygen tension, subcutaneous oxygen tension and gastrointestinal intramucosal $\mathrm{pH}$ in pigs. Crit Care Med 1991; 19: 205-10.

73. Arnold J, Hendricks J, Ince C, et al - Tonometry to assess the adequacy of splanchnic oxygenation in the critically ill patient. Intensive Care Med 1994; 20: 452-6.

74. Boda D, Murangi L - Gastrotonometry: and aid to the control of ventilation during artificial respiration. Lancet 1959; 273: 181-2.

75. Taylor DE, Gutierrez G - Tonometry. A review of clinical studies. Crit Care Clin 1996; 12: 1007-18.

76. Walley KR, Friesen BP, Humer MF, etal-Small bowel tonometry is more accurate than gastric tonometry in detecting gut ischemia. J Appl Physiol 1998; 85: 1770-7.

77. Janssens U, Groesdouk H, Graf J, Radke PW, et al - Comparison of esophageal and gastric air tonometry in patients with circulatory failure. Br J Anesth 2002; 89: $237-41$.

78. Myten MG, Webb AR - Intraoperative gut mucosal hypoperfusion is associated with increased postoperative complications and cost. Intensive Care Med 1994; 20: 99-104.

79. Londow L, Phillips DA, Heard SO, et al. - Gastric tonometry and venous oximetry in cardiac surgery patients. Crit Care Med 1991; 19: 1226-33.

80. Doglio GR, Pusajo JF, Egurrola MA, et al-Gastric mucosal $\mathrm{pH}$ as a prognostic index of mortality in critically ill patients. Crit Care Med 1991; 19: 1037-40.

81. Diebel L, Kazaol R, Wilson RF, et al - Gastric intramucosal acidosis in patients with chronic kidney failure. Surgery 1993; 113: 520-6.

82. Fiddian-Green RG, Baker S. Predictive value of the stomach wall $\mathrm{pH}$ for complications after cardiac operations: comparison with other monitoring. Crit Care Med 1987; 15: 153-6.

83. Gys T, Hubens A, Neels $\mathrm{H}$, et al-Prognostic value of gastric intramucosal $\mathrm{pH}$ in surgical intensive care patients. Crit Care Med 1988; 16: 1222-4.

84. Maynard N, Bihar D, Beale R, et al. - Assessment of splanchnic oxygenation by gastric tonometry in patients with acute circulatory failure. JAMA 1993; 270 : 1203-10.

85. Dagan O, O'Donnovan F, Cox P - An evaluation of gastric $\mathrm{pH}$ tonometry in neonates undergoing high-risk cardiac surgery. Crit Care Med 1993; 21: S148.

86. Wippermann CF, Schmid FX, Kampmann C, et al. - Evaluation of intramucosal pH during and after pediatric cardiac surgery. Eur J Cardiothorac Surg 1997; 12: 190-4.

87. Pérez A, Schnitzler EJ, Minces PG-The value of gastric intramucosal pH in the postoperative period of cardiac surgery in pediatric patients. Crit Care Med 2000; $28: 1585-9$.

88. Soybir N, Tekin S, Koner O, et al - Gastric tonometer monitoring in infants undergoing repair of coarctation of the aorta. J Cardiothorac Vasc Anesth 2000;14: 672-5.

89. Souza RL, Carvalho WB, Maluf MA, et al - Avaliação da perfusão esplâncnica com a utilização da tonometria gástrica no pós-operatório imediato de cirurgia cardíaca em crianças. Arq Bras Cardiol 2001; 77: 509-14.

90. Russell JA - Gastric tonometry: does it work? Intensive Care Med 1997; 23: 3-6.

91. Takala J, Parviainen I, Siloaho M, et al -Saline $\mathrm{P}_{\mathrm{CO} 2}$ is an important source of error in the assessment of gastric intramucosal pH. Crit Care Med 1994; 22: 1877-9.

92. Atik E-Cateterismo cardíaco intervencionista na cardiologia pediátrica. O posicionamento médico quanto às amplicações atuais e perspectivas. Arq Bras Cardiol 2002; 79: 443-5. 